\title{
Aerodynamic Analysis of Small Propeller in Wind Field Using CFD
}

\author{
Chuansheng Zhang ${ }^{1, \text { a }}$, Shengguang $\mathrm{Xie}^{2, \mathrm{~b}}$ and Tianhao Qin ${ }^{3, \mathrm{c}}$ \\ ${ }^{1}$ G615,New main building, Beijing university of aeronautics and astronautics, Beijing, China \\ ${ }^{2} \mathrm{G} 615$,New main building, Beijing university of aeronautics and astronautics, Beijing, China \\ ${ }^{3}$ Beijing university of science and technology, Beijing, China \\ achuansheng_zhang@yeah.net, bshengguangxie@gmail.com, 'aintianhaohao@163.com
}

\begin{abstract}
Keywords: propeller; aerodynamic; CFD; wind field
Abstract. In this paper, a propeller model which is common used in small multirotor aerial vehicles is created in CATIA software. To analysis the aerodynamic performance of the propeller in wind field, numerical simulation method in fluent software is used. Through the building of computational domain, meshing of the propeller and computational field and condition settings, the data of aerodynamic forces is collected. Then we analysis the relationship between propeller's forces, wind field and propeller's rotation speed. Some conclusions about the aerodynamic performance are drawn from the analysis results.
\end{abstract}

\section{Introduction}

Small propeller is widely used in small helicopters or multirotor aerial vehicles and the aerodynamic performance of propeller is essential to the aerial vehicle's efficiency,stability and flight safety. For a 10 inches propeller, it's Reynolds number in normal working condition is about $1 \times 10^{5}$ [1]. The analysis methods used in large scale rotary blade which neglecting the effects of air viscosity can not be applied to small propeller. What's more, when the propeller's air column subject to the wind disturbance, the propeller's aerodynamic performance becomes more complicated. In the anti-wind research of propeller based aerial vehicles, for the purpose of compensate wind disturbance effects, this paper studied the aerodynamic performance of small propeller in wind field using CFD numerical simulation software.

There has been some work on the analysis of propeller aerodynamic performance. Peter J. Kunz[2] developed a design method for ultra-low Reynolds number rotors which is usually below 10,000. This method is proved useful in the initial designs. Jin Jiang etc.[3] studied the aerodynamic performance and characteristic of aerofoil, blade and unit of wind turbine by the aerodynamic numerical simulation and performance prediction. Suwan Prak etc.[4] presented a dynamic model for calculating the forces due to the rotor blades colliding with air flows and the force equations suitable for the existing for the rigid-body simulation systems. Then the results could be used in real-time processing behavior. Haomiao Huang etc.[5] took blade flapping and thrust variation into consideration in the computation of blade's thrust. Through a feedback linearization controller, the two aerodynamic effects are compensated. Caitlin Powers etc.[6] proposed a similar model that considers rotor speed, vehicle velocity, blade pitch and angle of attack, variables that are difficult to estimate during flight.

In this paper, we take the common used propeller in multirotor aerial vehicles as the research object. To realize the rotation of propeller and apply the outer wind field disturbance conditions, we present a novel computational domain for the propeller. Through change the wind velocity and direction, we record the aerodynamic forces in three dimension with different propeller's rotation speed. By analysis the recording data, some useful conclusions are drawn and then used in the prototype design and wind disturbance compensate design of aerial vehicles.

The remainder of the paper is organized as follows: in the background works part, the propeller's model and computational domain are present. We also discuss the settings of relative conditions and the data we need to record in favor of aerodynamic analysis. In the experimental results analysis part, 
we analysis the propeller's aerodynamic characteristic and the results would be listed in the conclusions part.

\section{Background Works}

The propeller's physical model is just as the rotary blade used in the small multirotor aerial vehicle. Fig. 1 shows the physical model of the propeller used in the CFD analysis, which is created in the CATIA software and its diameter is 254 millimeter. The blade element shape of the propeller at radius $r$ is CLARK_Y. This type of propeller is usually used in multirotor aerial vehicles with four, six or eight rotors[7]. Through test with brush-less motor X2212, the actual rotation speed can reach to 7000RPM. To get the overall performance of the propeller, the rotation speed of the propeller is set to 1500 RPM, 2000 RPM, 2500 RPM, 3000 RPM, 3500 RPM, 4000 RPM, 4500 RPM, 5000 RPM, 5500 RPM, 6000 RPM, 6500 RPM and 7000 RPM.

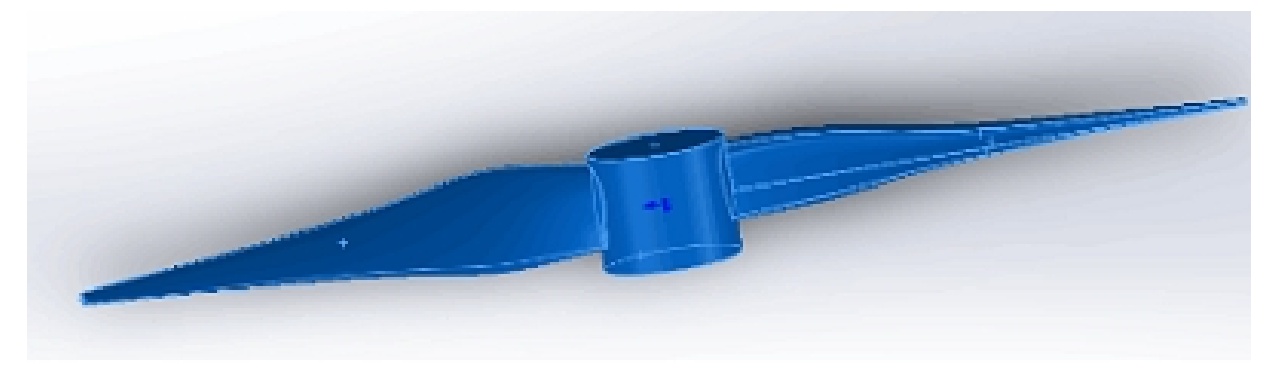

Fig.1. physical model of the propeller

To realize the movement of the propeller, we apply the sliding mesh technology to the design of computational domain[8]. More specifically, we create a cylinder containing the propeller model only just. As for the whole computational domain shape involving the overall airflow field, the idea come to our mind first is a large cylinder. But for the convenience of applying wind field condition, we replace the cylinder with a cuboid. Fig. 2 shows the setting of propeller's computational domain. The cylinder volume excluding the propeller entity is specified as a fluid continuum type, naming still. The cuboid excluding the cylinder volume is specified as a fluid continuum type, naming move. To realize the movement of propeller, we set the move field as moving mesh and set the rotation speed value as the desired propeller's rotation speed, while rotation speed of the propeller entity and the still field is set to zero. What calls for special attention is that the faces which are coincident between still field and move field should be set as interface. There are three pairs of interface in this work.

To analysis the aerodynamic performance of the propeller, wind field is applied to the propeller as the way shown in the right part of Fig.2. For a random wind field, we can decompose the wind vector to two direction, namely the direction perpendicular and parallel to the propeller's rotation plane. In this paper, we study the effects of vertical wind and horizontal wind respectively. For a multirotor aerial vehicle, the usually wind speed acting on the propeller is relatively small, and the maximum wind velocity in the simulation is set to $8 \mathrm{~m} / \mathrm{s}$. To compare the effect of wind velocity on propeller's aerodynamic performance, the vertical wind velocity and horizontal wind velocity are set to $2 \mathrm{~m} / \mathrm{s}, 4$ $\mathrm{m} / \mathrm{s}, 6 \mathrm{~m} / \mathrm{s}$ and $8 \mathrm{~m} / \mathrm{s}$ respectively. 


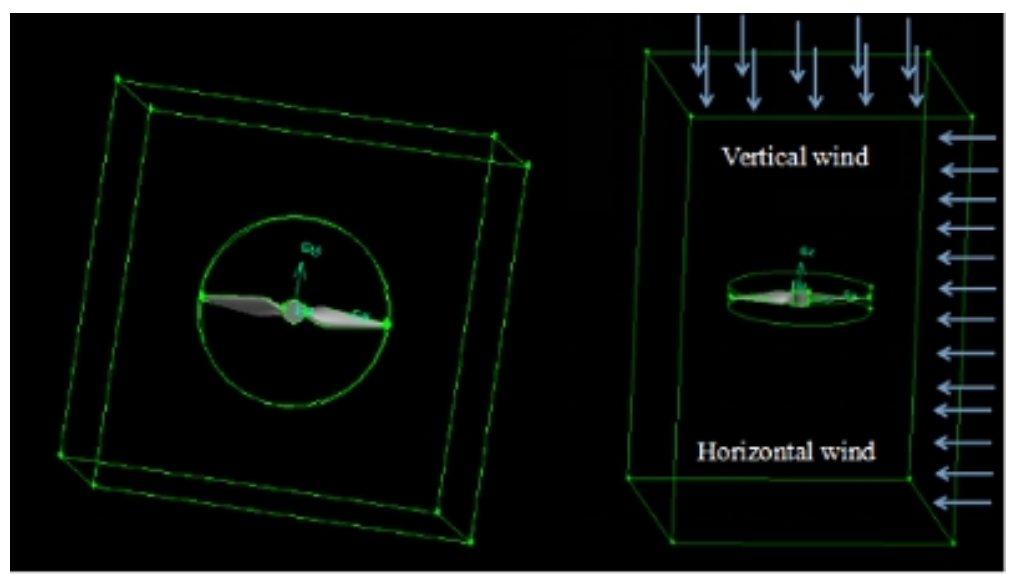

Fig.2. setting of propeller's computational domain. A fixed coordination system Oxyz is supposed, with its $\mathrm{O}$ point fixed in the geometry center of the propeller, and $\mathrm{Z}$ axis vertical to the propeller's rotary surface, $\mathrm{X}$ axis point to the right side surface expressed in the right part of Fig.2, and $\mathrm{Y}$ axis subject to right hand principle.

Before numerical simulation, the computational domain and propeller entity should be meshed. The mesh quality of the computational domain will affects the calculation accuracy and computational convergence speed directly. Firstly we mesh the propeller entity. The sequence we follow is from line to surface and then the body. Because the curvature of the propeller's surface is too bigger, some works should be done to reduce the curvature, including split and merge of the surface. Fig. 3 shows the result of meshing of the whole computational domain.

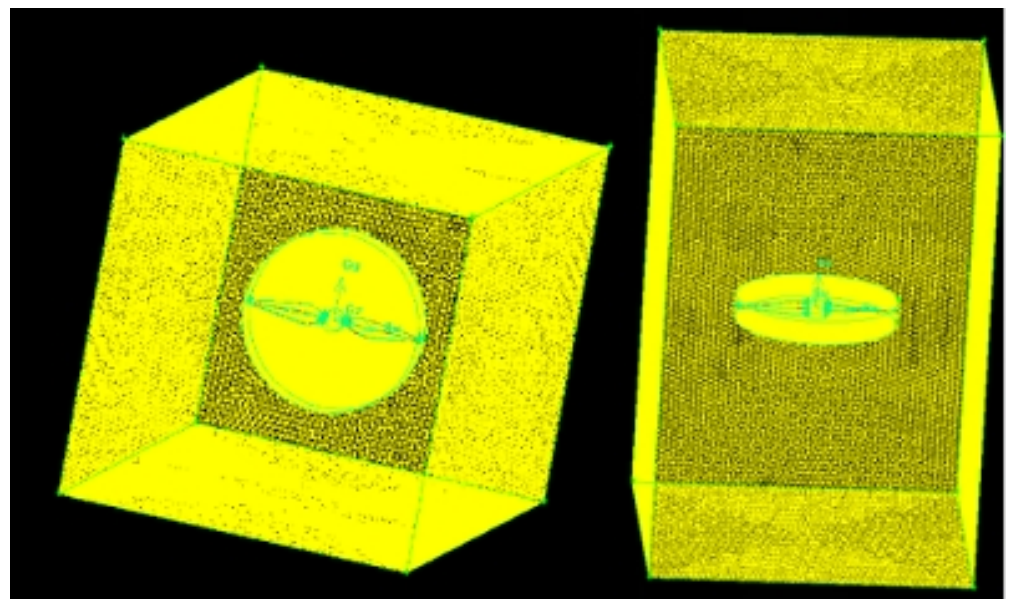

Fig.3. mesh of computational domain and propeller entity

Computational fluid dynamics (CFD) is a numerical simulation method to solver the propeller's three dimensional airflow field. Specifying the boundary conditions, turbulence model and physical parameters on meshing models, using the iterative method to solve the physical quantity on the grid nodes, we finally get the result until the residual error convergence[9]. Detached-Eddy simulation model is selected as the turbulence model. The residual error convergence precision is set to 0.001 . In the process of iteration, twenty steps equal to one second movement of the propeller.

\section{Experimental Results Analysis}

Fig.4 is the line graph of propeller's thrust performance when vertical wind is added. Two points can be drawn from the Fig.4.

(1) The propeller's thrust value decrease with the increase of vertical wind velocity. Through calculation, though not strict enough, the thrust decrease about 10 percent with the vertical wind increase $2 \mathrm{~m} / \mathrm{s}$. 
(2) We also have noticed that when the propeller's rotation speed equals to 6500 RPM and 7000 RPM, the propeller's thrust has a great gap between the condition of $\mathrm{Z}=0$ and others. What can we learn from the point is that we should avoid the propeller's speed equals to a speed above 6500 RPM when the aerial vehicles flying outdoors.

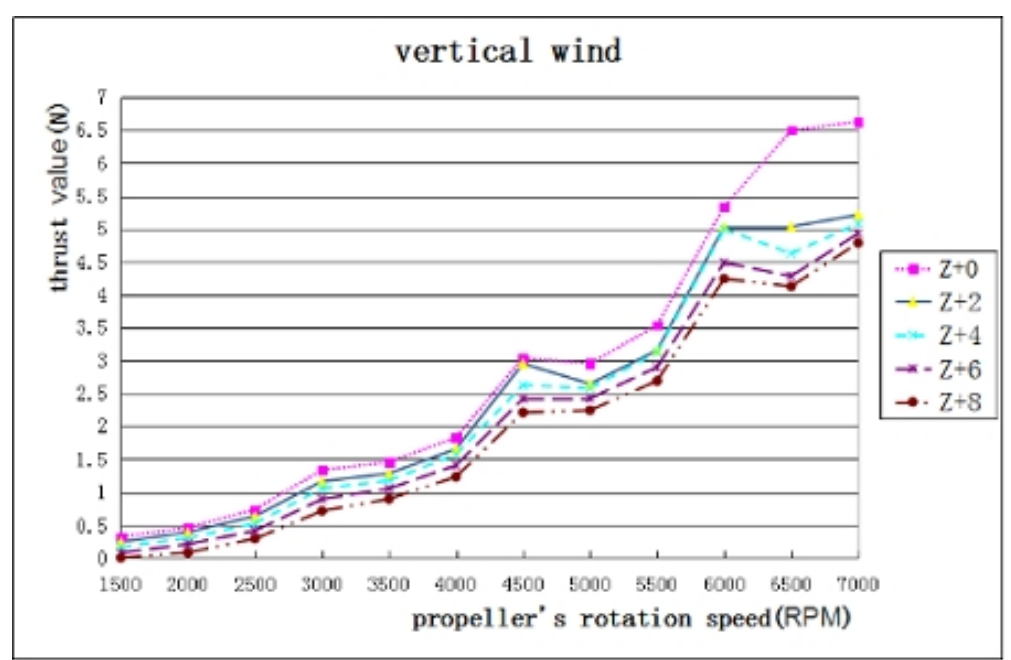

Fig.4. line graph of the propeller's performance when vertical wind is added. Where the $Z+2$ represent the wind comes from the positive side of $Z$ axis and the wind velocity is $2 \mathrm{~m} / \mathrm{s}$. $Z+0, Z+4, Z+6, Z+8$ conveys the same meaning. The point in the figure means the thrust value at certain rotation speed and vertical wind velocity.

Fig.5 is the line graph of propeller's thrust performance when horizontal wind is added. From Fig.5 we can see that:

(1)When the propeller's rotation speed is less than 6000 RPM, the propeller's thrust value has little to do with the horizontal wind. However, when the rotation speed equals to 6500 RPM and 7000 RPM, the thrust decrease greatly as long as there is horizontal wind field.

(2)Comparing the broken line of $\mathrm{X}+2, \mathrm{X}+4, \mathrm{X}+6, \mathrm{X}+8$, we can find that these lines are nearly superposition.

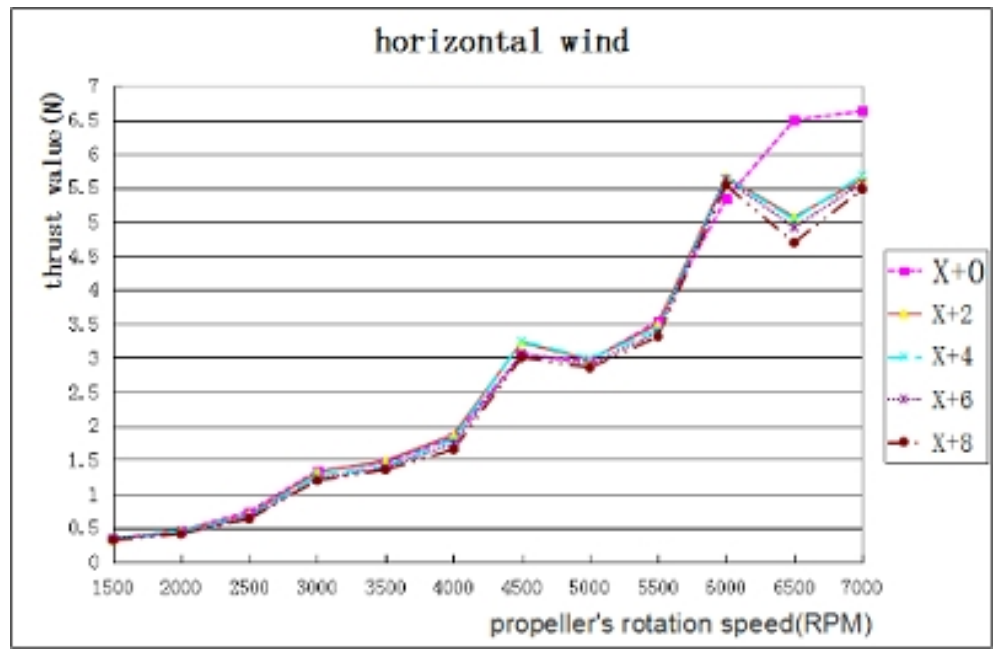

Fig.5. line graph of propeller's performance when horizontal wind is added. Where the $X+2$ represent the wind comes from the surface marked in Fig. 2 and the wind velocity is $2 \mathrm{~m} / \mathrm{s} . \mathrm{X}+0, \mathrm{X}+4, \mathrm{X}+6, \mathrm{X}+8$ represent the wind comes from the same surface. The point in the figure means the thrust value at certain rotation speed and horizontal wind velocity.

In the process of aerodynamic force collection, we find that the data including the aerodynamic force in three dimensional fluctuates similar to the sine wave. Fig.6 shows the propeller's thrust 
fluctuate over time when the rotation speed is 5500 RPM. As the increase of horizontal wind velocity, the amplitude of the fitted curve become large too. The feature has also been tested by other data. The thrust values expressed in Fig.4 and Fig.5 are got by computing the average value of peaks.

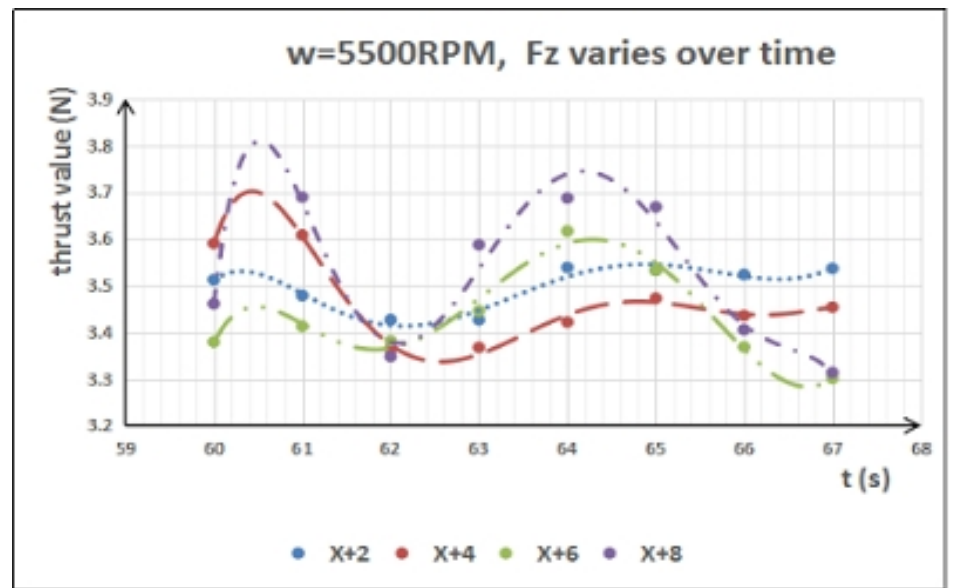

Fig.6. the propeller's thrust fluctuate over time when the rotation speed is 5500 RPM. The data is fitted by a six-degree polynomial curve. $\mathrm{w}$ represents the propeller's rotation speed.

In the following part, we discuss the aerodynamic force Fx which is parallel to the applied horizontal wind field. Imaging that a multirotor hovering in the sky, subjecting to the effects of horizontal wind, in order to keep the hovering position, the aerial vehicle need to eliminate the horizontal force. So the Fx force needed detailed analysis. Firstly we plot the line graph of Fx based on variable time. For a certain rotation speed, one figure is plotted. According to the characteristics of those figures, three classes are divided.

The first class involves propeller's rotation speed equals to 1500 RPM, 2000 RPM, 2500 RPM and 3000 RPM. For example, Fig.7 shows the aerodynamic force Fx varies over time, and the force value is generally less than 0 .

The second class involves propeller's rotation speed equals to 3500 RPM, 4000 RPM and 4500 RPM. For example, Fig. 8 shows the aerodynamic force Fx varies over time, and the force arises positive value, but the absolute value of negative peak is still bigger than the value of positive peak.

The third class involves propeller's rotation speed equals to 5000 RPM, 5500 RPM, 6000 RPM, 6500 RPM and 7000 RPM. For example, Fig.9 shows the aerodynamic force Fx varies over time, and the broken line is similar to the sine curve. The value of positive peak is nearly equals to the absolute value of negative peak.

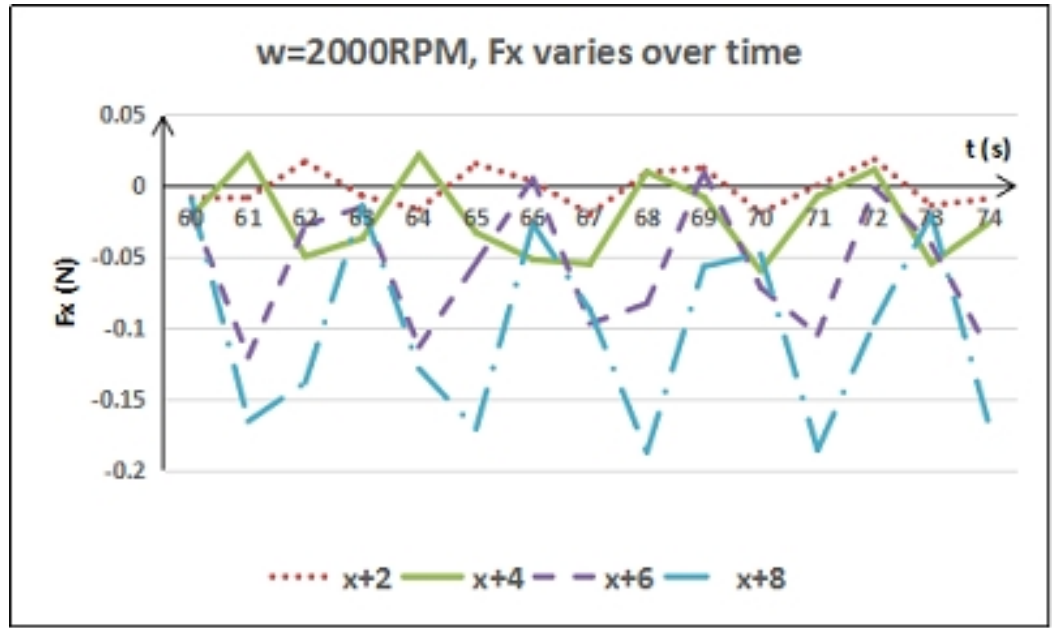

Fig.7. the aerodynamic force Fx varies over time when the rotation speed equals to 2000 RPM 


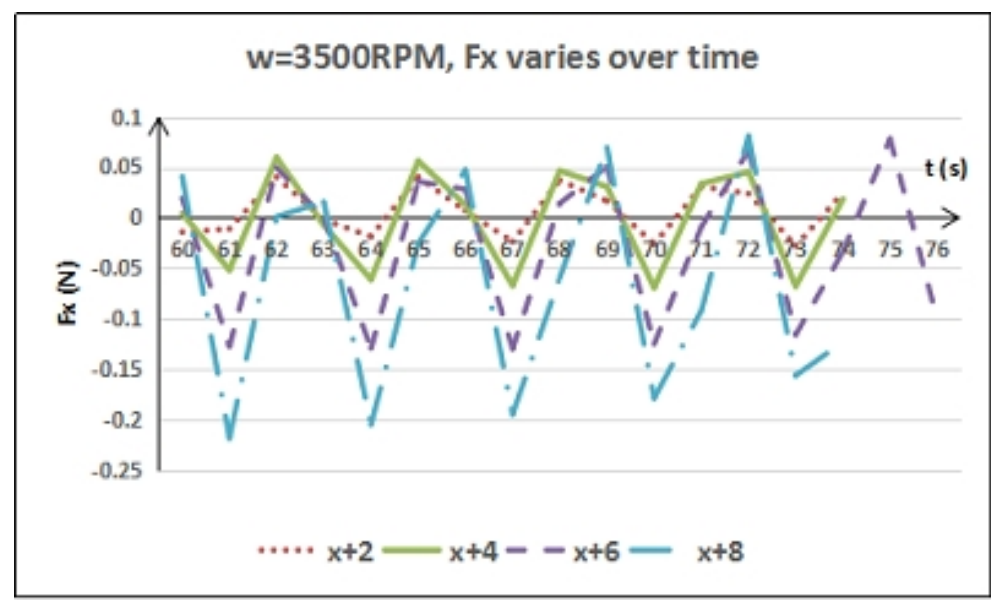

Fig.8. the aerodynamic force Fx varies over time when the rotation speed equals to 3500 RPM

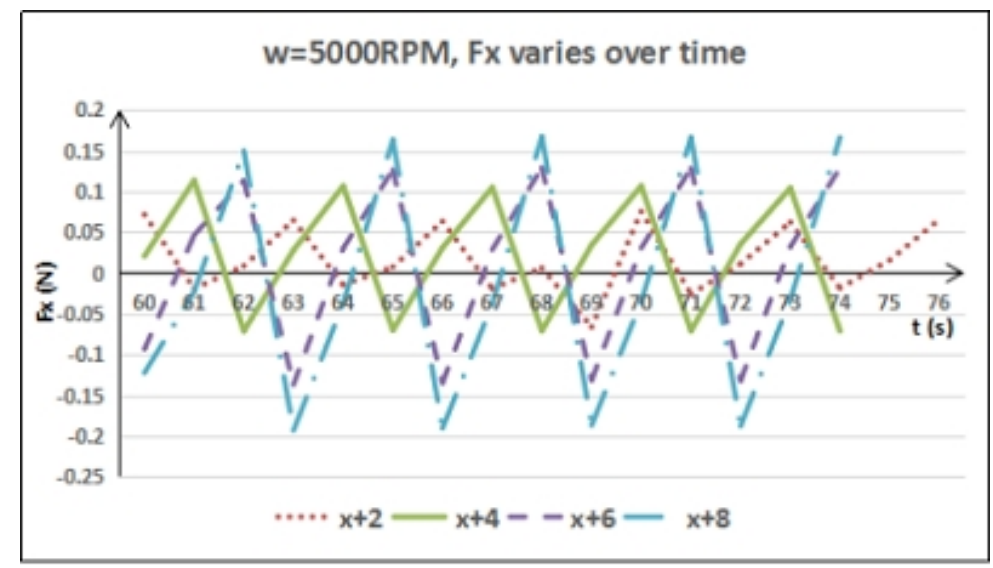

Fig.9. the aerodynamic force Fx varies over time when the rotation speed equals to 5000 RPM

Fig.10 shows the relationship between Fx and horizontal wind velocity at a certain propeller's rotation speed. The points of a certain rotation speed is fitted by straight line and the effect of the fitting is pretty good. This illustrates the linear relationship between aerodynamic force Fx and horizontal wind speed.

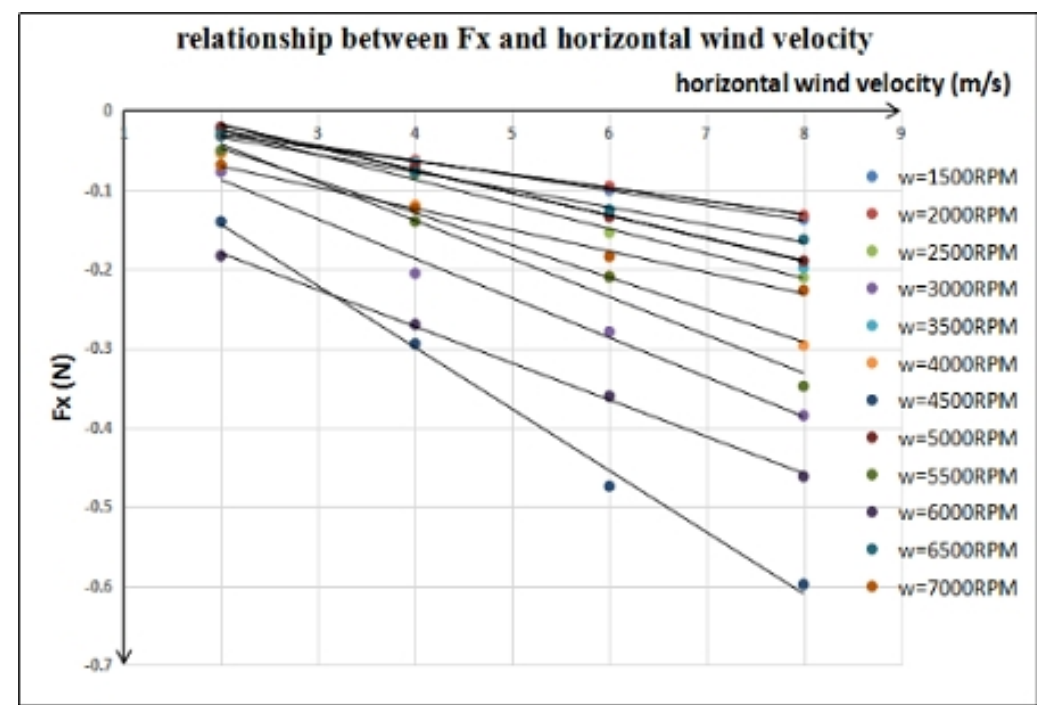

Fig.10. relationship between Fx and horizontal wind velocity. The value of every point expressed in the figure is acquired by averaging the force value of negative peaks at certain propeller's rotation speed and horizontal wind velocity. 
Table 1 presents the equations of the straight lines expressed in Fig.10. According to classes divided above, the equations are also divided into three classes. For the first class, the slope generally increases with the increase of propeller's rotation speed and this is also same to the second class. For the third class, the maximum slope of the lines comes when the propeller's rotation speed equals to 5000 RPM. The equations provide a method of horizontal aerodynamic force compensation and this is essential to the position control precision of multirotor aerial vehicles.

Table 1. equations between Fx and horizontal wind velocity $\mathrm{Vh}$

\begin{tabular}{|c|c|c|c|c|c|}
\hline $\begin{array}{c}\mathrm{W} \\
(\mathrm{RPM} \\
\text { ) }\end{array}$ & $\mathrm{Fx}(\mathrm{Vh})$ & $\begin{array}{c}\mathrm{W} \\
(\mathrm{RPM})\end{array}$ & $\mathrm{Fx}(\mathrm{Vh})$ & $\begin{array}{c}\mathrm{W} \\
(\mathrm{RPM})\end{array}$ & $\mathrm{Fx}(\mathrm{Vh})$ \\
\hline 1500 & $\begin{array}{l}\mathrm{Fx}=-0.01885^{*} \mathrm{Vh}+ \\
0.0125\end{array}$ & 3500 & $\begin{array}{l}\mathrm{Fx}=-0.02885 * \mathrm{Vh}+ \\
0.04055\end{array}$ & 5000 & $\mathrm{Fx}=-0.02849 * \mathrm{Vh}+0.0387$ \\
\hline 2000 & $\begin{array}{l}\mathrm{Fx}=-0.01676^{*} \mathrm{Vh}+ \\
0.0033\end{array}$ & 4000 & $\begin{array}{l}\mathrm{Fx}=-0.04079 * \mathrm{Vh}+ \\
0.0342\end{array}$ & 5500 & $\mathrm{Fx}=-0.04816^{*} \mathrm{Vh}+0.0539$ \\
\hline 2500 & $\begin{array}{l}\mathrm{Fx}=-0.03113 * \mathrm{Vh}+ \\
0.0376\end{array}$ & 4500 & $\begin{array}{l}\mathrm{Fx}=-0.0777 * \mathrm{Vh}+ \\
0.0118\end{array}$ & 6000 & $\begin{array}{l}\mathrm{Fx}=-0.04634 * \mathrm{Vh}- \\
0.08715\end{array}$ \\
\hline \multirow[t]{2}{*}{3000} & $\begin{array}{l}\mathrm{Fx}=-0.04986 * \mathrm{Vh}+ \\
0.01265\end{array}$ & & & 6500 & $\mathrm{Fx}=-0.02208 * \mathrm{Vh}+0.0107$ \\
\hline & & & & 7000 & $\begin{array}{l}\mathrm{Fx}=-0.02682 * \mathrm{Vh}- \\
0.01645\end{array}$ \\
\hline
\end{tabular}

Fig.11 shows the line graph between aerodynamic force Fx and propeller's rotation speed for different horizontal wind speed. From the figure, conclusions can be drawn that:

(1) The line has peak value obviously when the rotation speed equals to 3000 RPM, 4500RPM and 6000 RPM. From the point of anti-wind ability, peak value of horizontal aerodynamic force should be avoided.

(2) When the rotation speed equals to 4500 RPM, the thrust value and Fx are all having peak values. To utilize the point, a ducted propeller could be considered.

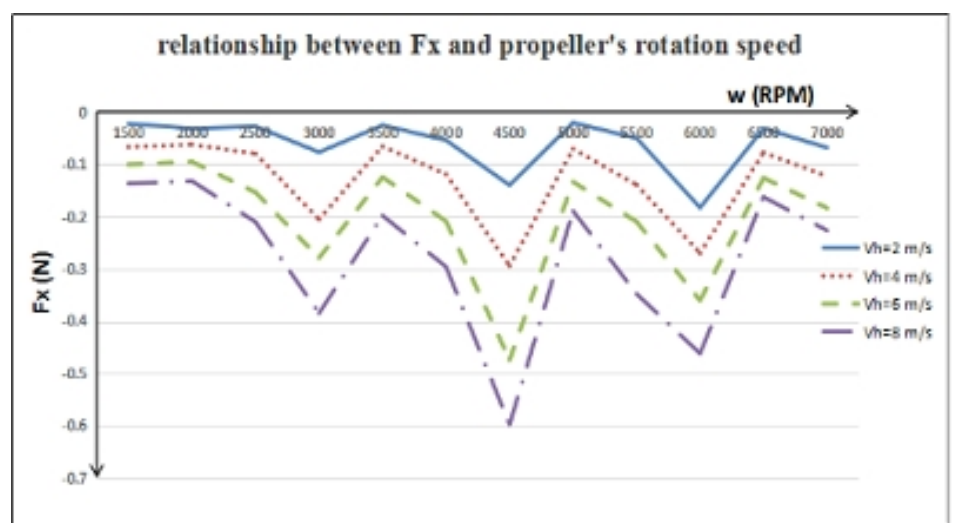

Fig.11. relationship between aerodynamic force Fx and propeller's rotation speed for a certain horizontal wind speed. 
From the above analysis, conclusions could be drawn that the aerodynamic performance of the propeller is pretty good when the rotation speed equals to $3500 \mathrm{RPM}, 4000 \mathrm{RPM}, 5000 \mathrm{RPM}$ and 5500 RPM. The rotation speed equals to 1500 RPM, 2000 RPM and 2500 RPM is neglected considering the load capacity in real flight.

Fig.12 listed the compensation results of each rotation speed when the horizontal wind speed equals to $4 \mathrm{~m} / \mathrm{s}, 6 \mathrm{~m} / \mathrm{s}$ and $8 \mathrm{~m} / \mathrm{s}$. There is no need for compensation when the horizontal wind speed equals to $2 \mathrm{~m} / \mathrm{s}$, for the horizontal force is pretty small. After compensation, the aerodynamic force turn into a curve similar to sine function. This illustrate that in the method of anti-wind research the aerial vehicle needs eliminating vibration even after the tilting compensation of horizontal force. We have also noticed that there is no angle tilting compensation at all, for the horizontal force similar to sine function.

The regulation of aerodynamic force Fy is similar to aerodynamic force Fx. In general, the propellers are layout symmetry and the aerodynamic forces in $\mathrm{Y}$ axis would be eliminated with each other.

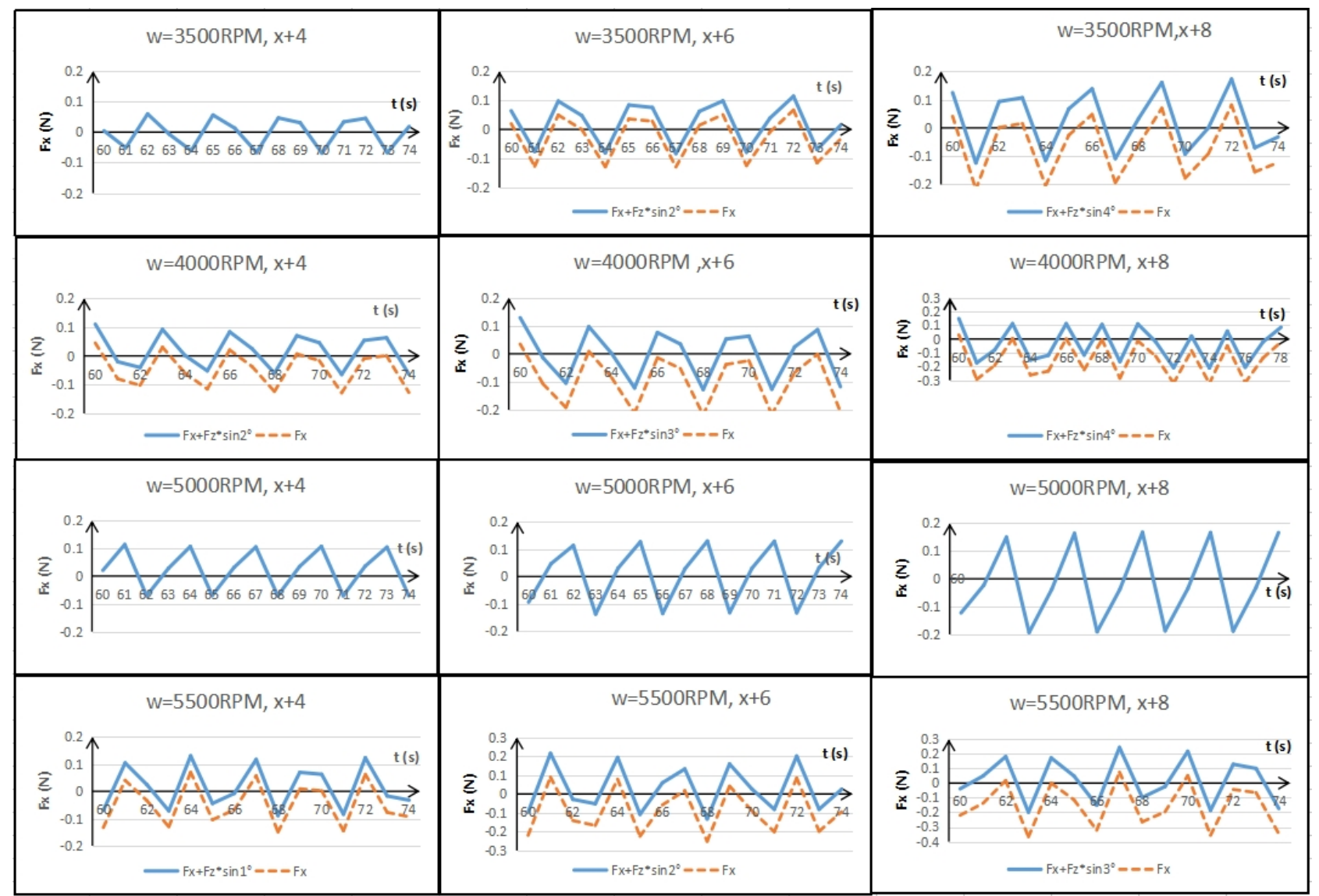

Fig.12. compensation of horizontal aerodynamic force when the propeller's rotation speed equals to 3500 RPM, 4000 RPM, 5000 RPM and 5500 RPM. The compensation angle is assumed to the positive when the rotation direction point to the positive side of $\mathrm{Y}$ axis.

\section{Conclusions}

From the analysis above, conclusions could be drawn safely that:

(1) The propeller's thrust value decrease with the increase of vertical wind velocity. Through calculation, though not strict enough, the thrust decrease about 10 percent with the vertical wind increase $2 \mathrm{~m} / \mathrm{s}$; 
(2) When the propeller's rotation speed is less than 6000 RPM, the propeller's thrust value has little to do with the horizontal wind. However, when the rotation speed equals to 6500 RPM and 7000 RPM, the thrust decrease greatly as long as there is horizontal wind field applied;

(3) The aerodynamic forces of the propeller change periodically over time just like sine function and its amplitude increases with the increase of wind speed;

(4) The aerodynamic performance of the propeller is better when the rotation speed equals to 3500 RPM, 4000 RPM, 5000 RPM and 5500 RPM. In the design of multirotor aerial vehicles, the rotation speed of hoving could be adjusted to those values;

(5) When the rotation speed equals to 4500 RPM, the aerodynamic forces of the propeller are all having peak values. To utilize the point, a ducted propeller could be considered;

(6) After angle tilting compensation for propeller's horizontal force, the aerodynamic force turn into a curve similar to the sine function which may cause vibration in the wind direction. For different propeller's rotation speed, the angle varied and has no certian relationship.

\section{References}

[1] B. R. Hein, I. Chopra, Hover performance of a micro air vehicle: rotors at low Reynolds number, published in proceedings of the American Helicopter Society International Specialists' meeting on Unmanned Rotorcraft, Chandler, AZ(2005)

[2] Peter J. Kunz, Roger C. Strawn, in: Analysis and design of rotors at ultra-low Reynolds numbers, AIAA, 2002-0099

[3] Jin Jiang, Zhihuai Xiao, Guoyu Zhang, Xiaohong Weng, in: Aerodynamic numerical simulation and performance prediction of wind turbine rotor, $\operatorname{IEEE}(2011)$

[4] Suwan Park, Nakhoon Beak, Kwan-Woo Ryo, in: A dynamic model of rotor blades for real-time helicopter simulation, submitted to Journal of Multimedia and Ubiquitous Engineering(2012)

[5] H. Huang, G. M. Hoffmann, S. L. Waslander, and C. J. Tomlin, in: Aerodynamics and control of autonomous quadrotor helicopter in aggressive maneuvering, submitted to Journal of Robotics and Automation(2009)

[6] C. Power, D. Mellinger, A. Kushleyev, B. Kothmann, and V. Kumar, in: Influence of aerodynamics and proximity effects in quadrotor flight, in Proceedings of the International Symposium on Experimental Robotics(2012)

[7] Moses Bangura, Hyon Lim, H. Jin Kim, in: Aerodynamic power control for multirotor aerial vehicles. Submitted to journal of IEEE International Conference on Robotics and Automation (ICRA), Hong Kong, China(2014)

[8] Junbo Zhou, Yang Liu, in: FLUENT 6.3 from introduction to the master in flow field analysis in Chinese, chapter 10, mechanical industry publishers(2011)

[9] Peiqing Liu, in: propeller theory and application in Chinese, beijing university of aeronautics and astronautics publishers(2006) 